\title{
A New Formulation for Dynamic Facility Layout Problem with the Locations of Input and Output Points within Unequal-sized Departments
}

\author{
Yujie Xiao, a , Yan Zheng ${ }^{2, b}$ and Peng $\mathrm{Li}^{2, \mathrm{c}}$ \\ 1Jiangsu Key Laboratory of Modern Logistics, School of Marketing and Logistic Management, \\ Nanjing University of Finance \& Economics, Nanjing, 210023, China; \\ ${ }^{2}$ College of Automobile and Traffic Engineering, Nanjing Forestry University, Nanjing, 210037, \\ China. \\ ayujiexiao@njue.edu.cn, byzheng_x@163.com, cdawan007@njfu.edu.cn
}

\begin{abstract}
Dynamic facility layout problem (DFLP) is to arrange departments within a planar for multiple periods where the material flows between departments may change due to the varying of market demands, with the objective of minimizing the sum of total material handling cost and rearrangement cost. This paper proposes a new formulation of DFLP which is more realistic than those for traditional dynamic layout problems in two ways. First, departments in the new formulation have unequal sizes and can be placed anywhere within a two dimensional planar rather than at a set of predetermined locations. Second, the new formulation simultaneously determines the layout of departments and the locations of input/output (I/O) points while most researchers tackle these two issues sequentially and separately. An example problem is well solved by using the proposed formulation.
\end{abstract}

Keywords: dynamic facility layout problem; unequal-sized departments; input/output points.

\section{Introduction}

Dynamic facility layout problem (DFLP) is to arrange departments within a planar for multiple periods where the material flows between departments may change due to the varying of market demands, with the objective of minimizing the sum of total material handling cost and rearrangement cost. Traditional DFLP is formulated based on the quadratic assignment problem (QAP), which assigns all departments to predetermined sites [1-3]. In those formulations of DFLP, departments are assumed to have equal sizes and input/output $(\mathrm{I} / \mathrm{O})$ point in each departments are assumed to be at the center of the predetermined sit. However, because the sizes, shapes, and the I/O locations of departments vary, the QAP-based formulation for DFLP has limitations in accurately reflecting real world cases. This paper presents a new formulation of DFLP where departments have unequal sizes and fixed shapes. Moreover, the proposed formulation of DFLP simultaneously determines the layout of departments and the locations of input/output (I/O) points.

Some researchers have present several different formulations for DFLP with unequal-sized departments $[4,5]$, but none of them deals with layout of departments and the locations of $\mathrm{I} / \mathrm{O}$ points simultaneously. This paper firstly explores the impact of relocation the I/O points on the layout rearrangement under dynamic environment. We attempt to discover an additional manner to respond to the dynamic market demand by actively modifying the locations of $\mathrm{I} / \mathrm{O}$ points beyond the general way of rearranging the departments.

\section{Mathematical Formulation for DFLP}

DFLP focused in this study is to simultaneously determine the positions of unequal size departments and the locations of I/O points of departments within a two dimensional planar for multiple periods. Each department has a rectangular shape and free orientations (i.e. departments can be placed horizontally or vertically) [6]. The amount of material flows from the output point to the input point for each pair of departments is given and may change during different time period in the planning horizon. Since the flows vary along the time horizon, an analysis of layout rearrangement 
during each pair of successive time periods is needed. The objective is to minimize the sum of the material handling cost (MHC) and department rearrangement cost (DRC) in the total planning horizon. MHC is defined as the product of the total travel distance (TTD) and the transportation cost per unit distance, where TTD is the sum of rectilinear distances between I/O points of departments weighted by the material flow quantities. The rearrangement cost for each department is also given as a constant number. To describe this DFLP, the notation is defined as follows.

Parameters

W Width of the two-dimensional planar.

$H \quad$ Height of the two-dimensional planar.

$N \quad$ Total number of departments.

$m, n \quad$ Indices for departments, $m=1, \ldots, N$ and $n=1, \ldots, N$.

$T \quad$ Total number of periods.

$t \quad$ Index of periods, $t=1, \ldots, T$.

$f_{m n t} \quad$ Material flow quantity from department $m$ to department $n(m \neq n)$ in period $t$.

$c \quad$ Cost for transferring a unit material a unit distance.

$d_{H m} \quad$ Half of the width of department $m$.

$d_{V m} \quad$ Half of the height of department $m$.

$R_{m t} \quad$ Rearrangement cost of department $m$ in period $t$.

Variables

$\left(x_{m t}^{I}, y_{m t}^{I}\right)$ Coordinates of input point of department $m$ in period $t$.

$\left(x^{O}{ }_{m t}, y^{O}{ }_{m t}\right)$ Coordinates of output point of department $m$ in period $t$.

$d^{X}{ }_{m n t}, y^{Y}{ }_{m n t}$ rectilinear distance form output point of department $m$ to input point of department $n$ in $x$-direction and $y$-direction in period $t$, respectively.

$\left(x_{m t}, y_{m t}\right) \quad$ Coordinates of the centre of department $m$ in period $t$.

$d_{X m t} \quad$ Horizontal distance from the centre of department $m$ to the boundary of department $m$ in period $t$.

$d_{Y m t} \quad$ Vertical distance from the centre of department $m$ to the boundary of department $m$ in period $t$.

$O_{m t}=\left\{\begin{array}{l}1, \text { if department } m \text { is placed horizontally, } \\ 0, \text { otherwise. }\end{array}\right.$

$w_{m n t}=\left\{\begin{array}{l}1, \text { if department } m \text { is placed to west of department } n \text { in } x \text {-direction in period } t \\ 0, \text { otherwise. }\end{array}\right.$

$s_{m n t}=\left\{\begin{array}{l}1, \text { if department } m \text { is placed to the south of department } n \text { in } y \text {-direction in period } t, \\ 0, \text { otherwise. }\end{array}\right.$

$r_{m t}=\left\{\begin{array}{l}1, \text { if department } m \text { is rearranged in period } t, \\ 0, \text { otherwise. }\end{array}\right.$

A nonlinear MIP model is presented for the proposed DFLP.

Minimize

$\sum_{t=1}^{T} \sum_{m, n=1}^{N} c f_{m n t}\left(d_{m n t}^{X}+d_{m n t}^{Y}\right)+\sum_{t=2}^{T} \sum_{m=1}^{N} R_{m t} r_{m t}$

Subject to

$$
\begin{aligned}
& d_{m n t}^{X}=\left|x_{m t}^{O}-x_{n t}^{I}\right| \quad \forall t, m, n, \text { and } m \neq n \\
& d_{m n t}^{Y}=\left|y_{m t}^{O}-y_{n t}^{I}\right| \quad \forall t, m, n, \text { and } m \neq n \\
& x_{m t}-d_{X m t} \leq x_{m t}^{O} \leq x_{m t}+d_{X m t} \quad \forall m, t \\
& y_{m t}-d_{Y m t} \leq y_{m t}^{O} \leq y_{m t}+d_{Y m t} \quad \forall m, t
\end{aligned}
$$




$$
\begin{aligned}
& x_{m t}-d_{X m t} \leq x_{m t}^{I} \leq x_{m t}+d_{X m t} \quad \forall m, t \\
& y_{m t}-d_{Y m t} \leq y_{m t}^{I} \leq y_{m t}+d_{Y m t} \quad \forall m, t \\
& d_{X m t}=d_{H m} O_{m t}+d_{V m}\left(1-O_{m t}\right) \forall m, t \\
& d_{Y m t}=d_{H m}\left(1-O_{m t}\right)+d_{V m} O_{m t} \quad \forall m, t \\
& d_{X m t} \leq x_{m t} \leq W-d_{X m t} \quad \forall m, t \\
& d_{Y m t} \leq y_{m t} \leq H-d_{Y m t} \quad \forall m, t \\
& x_{m t}+d_{X m t} \leq x_{n t}-d_{X n t}+W\left(1-w_{m n t}\right) \quad \forall m \neq n, t \\
& y_{m t}+d_{Y m t} \leq y_{n t}-d_{Y n t}+H\left(1-s_{m n t}\right) \quad \forall m \neq n, t \\
& w_{m n t}+w_{n m t}+s_{m n t}+s_{n m t}=1 \quad \forall m<n, t \\
& x_{m t}-x_{m, t-1} \leq W r_{m t} \quad \forall m, t>1 \\
& x_{m, t-1}-x_{m t} \leq W r_{m t} \quad \forall m, t>1 \\
& y_{m t}-y_{m, t-1} \leq H r_{m t} \quad \forall m, t>1 \\
& y_{m, t-1}-y_{m t} \leq H r_{m t} \quad \forall m, t>1 \\
& O_{m t}-O_{m, t-1} \leq r_{m t} \quad \forall m, k, t>1 \\
& O_{m, t-1}-O_{m t} \leq r_{m t} \quad \forall m, k, t>1 \\
& x_{m t}^{o}-x_{m, t-1}^{o} \leq W r_{m t} \quad \forall m, t>1 \\
& x_{m, t-1}^{O}-x_{m t}^{O} \leq W r_{m t} \quad \forall m, t>1 \\
& y_{m t}^{O}-y_{m, t-1}^{O} \leq H r_{m t} \quad \forall m, t>1 \\
& y_{m, t-1}^{O}-y_{m t}^{O} \leq H r_{m t} \quad \forall m, t>1 \\
& x_{m t}^{I}-x_{m, t-1}^{I} \leq W r_{m t} \quad \forall m, t>1 \\
& x_{m, t-1}^{I}-x_{m t}^{I} \leq W r_{m t} \quad \forall m, t>1 \\
& y_{m t}^{I}-y_{m, t-1}^{I} \leq H r_{m t} \quad \forall m, t>1 \\
& y_{m, t-1}^{I}-y_{m t}^{I} \leq H r_{m t} \quad \forall m, t>1 \\
& O_{m t}, w_{m n t}, s_{m n t} \in\{0,1\} \quad \forall k, t, m, n \text {, and } m \neq n \\
& x_{m t}^{I}, y_{m t}^{I}, x_{m t}^{O}, y_{m t}^{O}, x_{m t}, y_{m t}, d_{X m t}, d_{Y m t} \geq 0 \quad \forall m, t
\end{aligned}
$$

The objective function (1) is to minimize MHC and DRC. Constraints (2) and (3) denote the rectilinear distance from the output point of department $m$ to the input point of department $n$ in period $t$. Constraints (4)-(7) determine the coordinates of $\mathrm{I} / \mathrm{O}$ points for each department in period $t$. Constraints (8)-(11) determine the locations of all departments within the two-dimensional planar in period $t$. Constraints (12)-(14) prohibit overlap between departments, which are similar to constraints presented in Sherali et al. [7]. Constraints (15)-(28) ensure that the centroids, orientations, input points and output points of not rearranged departments have the same values. Constraint (29) restricts 0-1variables and Constraint (30) ensures the non-negativity of variables.

\section{Computational Experiments}

The MIP model is solved by CPLEX 9.0 and computational experiments are executed to test the effectiveness of the proposed model. The data of example problem with five departments is shown in 
Table 1. In Table 1, the material flow quantities between each of departments in period $1\left(f_{m n 1}\right)$ and period $2\left(f_{m n}\right)$ are listed. The sizes of the five departments are also listed in Table 1.

Table 1 Data of the example problem

\begin{tabular}{cccccc|ccccc|cc}
\hline & \multicolumn{9}{c|}{$f_{m n 1}$} & \multicolumn{7}{c|}{$f_{m n 2}$} & \multirow{2}{*}{$d_{H m}$} & \multirow{2}{*}{$d_{V m}$} \\
\cline { 2 - 10 } & 1 & 2 & 3 & 4 & 5 & 1 & 2 & 3 & 4 & 5 & \\
\hline 1 & 0 & 6 & 10 & 2 & 10 & 0 & 0 & 12 & 1 & 3 & 2.5 & 2 \\
2 & 0 & 0 & 17 & 15 & 15 & 0 & 0 & 19 & 1 & 10 & 4.5 & 4.0 \\
3 & 0 & 0 & 0 & 6 & 4 & 0 & 0 & 0 & 14 & 9 & 3.0 & 2.5 \\
4 & 0 & 0 & 0 & 0 & 11 & 0 & 0 & 0 & 0 & 11 & 3.0 & 2.0 \\
5 & 0 & 0 & 0 & 0 & 0 & 0 & 0 & 0 & 0 & 0 & 2.0 & 2.0 \\
\hline
\end{tabular}

To investigate the performance of simultaneous determination of the layout of departments and the I/O point locations, two experiments are conducted. The first one only consider the dynamic layout design of the five departments in two periods. The result is shown in Fig. 1 with total cost 1353, where MHC is 1334 and DRC is 19. Department 4 and 1 are rearranged. The other one simultaneously determines the layout of departments and the locations of I/O points. The layout solution with total cost 352 is depicted in Fig. 2. As shown in Fig. 2, we may beyond the general way of rearranging the departments by actively modifying the locations of $\mathrm{I} / \mathrm{O}$ points.

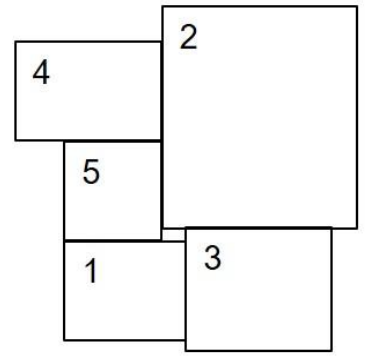

(a) layout in period 1

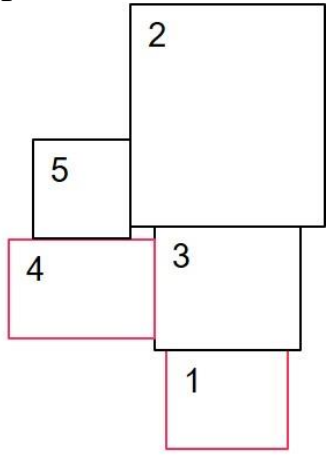

(b) layout in period 2

Fig. 1 Layout design without consideration of locations of $\mathrm{I} / \mathrm{O}$ points

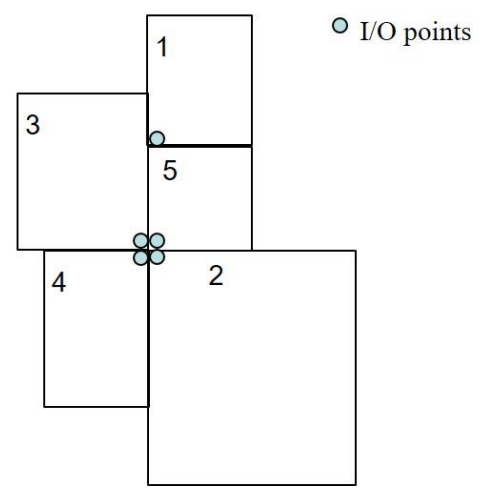

Fig. 2 Layout design with consideration of locations of I/O points

\section{Conclusion}

This paper deals with the DFLP with the Locations of Input and Output Points within Unequal-sized Departments. A new MIP formulation is presented to solve DFLPs optimally. This paper firstly explores the impact of relocation the $\mathrm{I} / \mathrm{O}$ points on the layout rearrangement under dynamic environment. Computational experiments show that simultaneously determination of the layout of departments and the locations of $\mathrm{I} / \mathrm{O}$ points may be an additional manner to respond to the dynamic market demand by actively modifying the locations of I/O points beyond the general way of rearranging the departments. 


\section{Acknowledgements}

The research is supported by the National Natural Science Foundation of China (Grant No. 71501090, 61403204), the Natural Science Foundation of Jiangsu Higher Education Institutions of China (Grant No. 14KJD410001).

\section{References}

[1] Rosenblatt, M.J. The dynamics of plant layout. Management Science. Vol. 32 (1986) No. 1, p. 76-86.

[2] Balakrishnan, J., Cheng, C.H. Dynamic layout algorithms: A state-of-the-art survey. Omega. Vol. 26 (1998) No. 4, p. 507-521.

[3] Kulturel-Konak, S. Approaches to uncertainties in facility layout problems: Perspectives at the beginning of the 21st century. Journal of Intelligent Manufacturing. Vol. 18 (2007) No. 2, p. 273-284.

[4] Yang, t., Peters, B.A. Flexible machine layout design for dynamic and uncertain production environments. European Journal of Operational Research. Vol. 108 (1998) No. 1, p. 49-64.

[5] Dunker, T., Radons, G., Westkamper, E. Combining evolutionary computation and dynamic programming for solving a dynamic facility layout problem. European Journal of Operational Research. Vol. 165 (2005) No.1, p. 55-69.

[6] Xiao, J., Seo, Y., Seo, M. A two-step heuristic algorithm for layout design of unequal-sized facilities with input/output points. International Journal of Production Research. Vol. 51 (2013) No. 14, p. 4200-4222.

[7] Sherail, H.D., Fraticelli, B.M.P., Meller, R.D. Enhanced model formulations for optimal facility layout. Operations Research, Vol. 51(2003), No. 4, 629-644. 